\title{
Distributed Detection with Data Fusion for Malfunction Detection and Isolation in Fault Tolerant Flight Control Computers
}

\author{
Celeste M. Belcastro \\ NASA Langley Research Center \\ Mail Stop 130 \\ Hampton, VA 23681-0001 \\ Phone: (757) 864-6182 \\ Fax: (757) 864-4234 \\ celeste.m.belcastro@larc.nasa.gov
}

\author{
Bernice Weinstein \\ NASA Langley Research Center \\ Mail Stop 130 \\ Hampton, VA 23681-0001 \\ Phone: (757) 864-6193 \\ FAX: (757) 864-4234 \\ b.weinstein@larc.nasa.gov
}

\begin{abstract}
It is anticipated that electromagnetic field disturbances will be increasingly problematic for future aircraft control systems, which will perform numerous flight-critical functions. Shielding within the digital systems cannot eliminate these common-cause disturbances, and redundancy is ineffective in accommodating them. This paper presents a distributed detection scheme with data fusion for monitoring the function of redundant processing channels of a flight critical control system during operation. The detection scheme is demonstrated using data collected during closed-loop High Intensity Radiated Fields (HIRF) system effects experiments on a quad-redundant Flight Control Computer (FCC), which is executing Autoland control laws under flight conditions with heavy clear air turbulence. The performance of the monitoring system is assessed. The detection scheme presented in this paper can also be applied to redundancy management in fault tolerant systems.
\end{abstract}

\subsection{Introduction}

Future generations of advanced aircraft will require high-integrity flight-critical systems for adaptive guidance and control, integrated vehicle health management, stability augmentation, flutter suppression, and air flow control for optimized aerodynamic performance over the flight envelope. It is anticipated that these vehicles will require a large number of distributed redundant processing nodes. Redundant processing elements will be used to handle massive data throughput and to mask random independent component failures. However, redundancy does not mask the effects of common-cause disturbances, such as lightning and HIRF that can affect the functional integrity of all processing elements without causing component damage. Moreover, it is not possible to completely eliminate the effects of these disturbances in the long term through protective means such as shielding. Laboratory experiments show that control computers that are subjected to electromagnetic disturbances can malfunction and cause catastrophic departures in performance of the closed-loop system [1] - [2].

The integrity of fault tolerant control computers in critical applications can be viewed at various levels of operation: system-level operation of controller functions such as redundancy management decisions, control law calculations, and input/output (I/O) rate/range checks [3]. This paper is concerned with the design and evaluation of a control law calculation malfunction detector. The design strategy for this detector was initially developed and analyzed using a simulation of the controller computations, as reported in [3] - [4]. An improvement to the design of the detector threshold was presented in [5]. The use of linear parameter varying models in the detector design was considered in [6], but will not be utilized in this paper. Other attempts to detect malfunctions in a fault tolerant flight control computer used a centralized detection scheme at the voted output of the controller [7]. Additional work in this area includes modeling and analysis of HIRF effects on flight controllers [8] - [9], as well as analysis of design tradeoffs for recoverable computing systems [10].

This paper presents the first attempt to apply the distributed control law calculation malfunction detection scheme to experimental data. The design applies distributed detection techniques with data fusion to monitoring the integrity of a flight control computer with redundant processing elements. An extensive literature review on fault detection and decision fusion methods is given in [11] - [12]. The approach presented herein is a hierarchical three-stage detection scheme. Malfunctions in the fault tolerant controller are detected by fusing the decisions from the monitors for $\mathrm{N}$ processors. Malfunctions in each processor are detected by fusing the decisions from $\mathrm{M}$ control law calculation monitors, one for each different control command. Malfunctions in each control law calculation are determined using the residual between a measurement of the calculation from the controller and an estimate of the correct calculation of the nominal (no malfunction) controller.

Section 2 presents a review of the problem formulation for the detector, and the design of the detector is presented in Section 3. Note that more general formulations for the detection problem and local detector threshold are presented here than those presented in [3] - [5]. In Section 4 , the monitoring strategy is demonstrated for the elevator and throttle commands of a Boeing 737 Autoland flight controller using data from closed-loop laboratory experiments in which a quad-redundant flight control computer was exposed to HIRF while executing the 
approach and landing. A summary and concluding remarks are given in Section 5.0.

\subsection{Problem Formulation}

Malfunction is defined as follows, in terms of monitoring the integrity of the control law calculations:

DEFINITION 1: The jth control law calculation of the ith processor at time step $\mathrm{k}$ is the result of a malfunction if:

$$
\left|\Delta \mathrm{z}_{\mathrm{i}}^{\mathrm{j}}(\mathrm{k})\right|>\varepsilon_{\mathrm{i}}^{\mathrm{j}}(\mathrm{k}) \text { for } \mathrm{k}=1, \ldots, \mathrm{K}
$$

$\Delta z_{j}^{j}(k)=$ change in the measurement of the $j$ th control law calculation of the ith processor at time step $k$ due to malfunction

$\varepsilon_{\mathrm{i}}^{\mathrm{j}}(\mathrm{k})=$ maximum allowable variation of $\mathrm{z}_{\mathrm{i}}^{\mathrm{j}}(\mathrm{k})$

$H=$ absolute value

The change $\Delta z_{i}^{j}(k)$ in the calculation of the $j$ th control law of the ith processor due to malfunction is defined as:

$$
\Delta z_{i}^{j}(k)=z_{i}^{j}(k)-E\left[z_{i}^{j}(k) \mid Z_{i}^{j}{ }^{k-1}\right]
$$

$z_{i}^{j}(k)=j$ th control law calculation of the ith processor at time step $\mathrm{k}$ which may reflect a malfunction

$z_{\mathrm{i}}^{\mathrm{j}}(\mathrm{k})=$ correct (no malfunction) control law calculation $\mathrm{j}$ of the ith processor at time step $\mathrm{k}$ $Z_{\mathrm{i}}^{\mathrm{j}^{\mathrm{k}-1}}=$ set of all past $z_{\mathrm{i}}^{\mathrm{j}}(\mathrm{k})$ up to time step $\mathrm{k}-1$

$E\left[z_{i}^{j}(k) \mid Z_{i}^{j^{k-1}}\right]=$ conditional expectation of correct (no malfunction) control calculation $\mathrm{j}$ at time step $\mathrm{k}$ given all past $z_{\mathrm{i}}^{\mathrm{j}}(\mathrm{k})$ up to time $\mathrm{k}-1$

DEFINITION 2: The ith processor in a fault tolerant control computer is malfunctioning if equation (1) holds for any $\mathrm{j}$ in $\{1, \ldots, \mathrm{M}\}$.

DEFINITION 3: The fault tolerant control computer is malfunctioning if equation (1) holds for any $i$ in $\{1, \ldots, N\}$.

Definition 3 considers the most conservative case in detecting malfunctions. By this definition, the fault tolerant controller is malfunctioning if any of the processors is malfunctioning. This is because the reliability of the fault tolerant computer is degraded if even one processor is malfunctioning. Other definitions of malfunction in the fault tolerant controller could also be considered.

The command calculation, $z_{\mathrm{i}}^{\mathrm{j}}(\mathrm{k})$, in equation (2) is defined as:

$$
z_{i}^{j}(k)=\vec{C}_{i}^{j} \vec{x}_{i}^{j}(k)+\vec{D}_{i}^{j} \vec{u}_{i}^{j}(k)+v_{i}^{j}(k)
$$

$\mathrm{z}_{\mathrm{i}}^{\mathrm{j}}(\mathrm{k})=$ measurement of $\mathrm{jth}$ control command from controller $i ; \quad \mathrm{z}_{\mathrm{i}}^{\mathrm{j}}(k) \in R ; \quad \mathrm{i}=1, \ldots, \mathrm{N} ;$ $\mathrm{j}=1, \ldots, \mathrm{M}$

$\vec{x}_{i}^{j}(k)=$ state vector for the $j$ th control command calculation from controller $i, \overrightarrow{\mathrm{x}}_{\mathrm{i}}^{\mathrm{j}}(\mathrm{k}) \in R^{p}$, $\mathrm{i}=1,2, \ldots, \mathrm{N} ; \mathrm{j}=1, \ldots, \mathrm{M}$

$\overrightarrow{\mathbf{u}}_{\mathrm{i}}^{\mathrm{j}}(\mathrm{k})=$ input vector for the $\mathrm{jth}$ control command calculation from controller $i, \overrightarrow{\mathrm{u}}_{\mathrm{i}} \mathrm{j}_{(\mathrm{k}) \in R^{L}}$ $v_{i}^{j}(k)=$ measurement noise for the jth control command of controller $i, v_{i}^{j}(\mathrm{k}) \in R$

$\overrightarrow{\mathrm{C}}_{\mathrm{i}}^{\mathrm{j}}=$ measurement weighting coefficient matrix for control law calculation $\mathrm{j}$ of controller $i$

$\overrightarrow{\mathrm{D}}_{\mathrm{i}}^{\mathrm{j}}=$ input weighting coefficient matrix for control law calculation $\mathrm{j}$ of controller $i$

The state vector is given by:

$$
\begin{aligned}
& \vec{x}_{i}^{j}(k+1)=\vec{A}_{i}^{j} \vec{x}_{i}^{j}(k)+\vec{B}_{i}^{j} \vec{u}_{i}^{j}(k)+\vec{\zeta}_{i}^{j} \vec{w}_{i}^{j}(k) \\
& \vec{w}_{\mathrm{i}}^{j}(\mathrm{k})=\text { process noise vector for the } \mathrm{jth} \text { control law } \\
& \text { calculation of controller } i ; \overrightarrow{\mathrm{w}}_{\mathbf{i}} \mathrm{j}_{(\mathrm{k}) \in R^{p}} \\
& \vec{\zeta}_{\mathrm{i}}^{\mathrm{j}}=\text { weighting matrix for the process noise vector } \\
& \overrightarrow{\mathrm{A}}_{\mathrm{i}}^{\mathrm{j}}=\text { state transition matrix for control law } \\
& \text { calculation } \mathrm{j} \text { of controller } i \\
& \vec{B}_{i}^{j}=\text { input weighting coefficient matrix for control } \\
& \text { law calculation } j \text { of controller } i \\
& k=\text { time step }
\end{aligned}
$$

The process noise $\vec{w}_{i}^{j}(k)$ in equation (4) accounts for modeling error, noise in the input vector $\overrightarrow{\mathrm{u}}_{\mathrm{i}}^{\mathrm{j}}(\mathrm{k})$ from the plant, and stochastic variations in the command that result 
from exogenous disturbances such as HIRF. Measurement noise $v_{i}^{j}(k)$ can also be affected by HIRF.

The initial state of the jth command calculation of the ith controller is denoted as $\overrightarrow{\mathrm{x}}_{\mathrm{i}}^{\mathrm{j}}\left(\mathrm{k}_{0}\right)$.

ASSUMPTION 1: The initial state $\vec{x}_{\mathrm{i}}^{\mathrm{j}}\left(\mathrm{k}_{0}\right)$ is a Gaussian

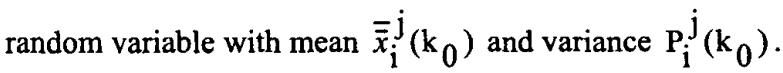
The initial state of the calculations of the ith controller are independent.

ASSUMPTION 2: The process noise $\vec{w}_{i}^{j}(k)$ is zero-mean, Gaussian, and white with variance $Q_{i}^{j}$. The process noise of the calculations of the ith controller are independent. Process noise $\bar{w}_{i}^{j}(k)$ is independent of the initial state $\bar{x}_{\mathrm{i}}^{\mathrm{j}}\left(\mathrm{k}_{0}\right)$.

ASSUMPTION 3: Measurement noise $v_{i}^{j}(k)$ is zero mean, Gaussian, and white with covariance matrix $R_{i}^{j}$. The measurement noise of the calculations of the ith controller are independent.

ASSUMPTION 4: Measurement noise $v_{i}^{j}(k)$ is statistically independent of the initial state $\left.\vec{x}_{i} \mathrm{j}_{0}\right)$ and the process noise $\vec{w}_{i}^{j}(k)$.

ASSUMPTION 5: Errors in the control law calculations, each of the processors, and the controller are independent.

\subsection{Multi-Stage Distributed Detector Design}

The malfunctions to be detected are defined by Definition 1. Detection of the phenomenon in each of these definitions is binary and can, therefore, be defined in terms of the general hypotheses:

$$
\begin{gathered}
\mathrm{H}_{1} \text { : Malfunction Condition } \\
\mathrm{H}_{0} \text { : Nominal (No Malfunction) Condition }
\end{gathered}
$$

The calculations of the controller are observed via noisy measurements $\mathrm{z}_{\mathrm{i}}^{\mathrm{j}}(\mathrm{k})$ with probability density $p\left[z_{i}^{j}(k) \mid H q\right], q=0,1$, from each of the processors.

3.1 Malfunction Detector for the jth Control Law Calculation of Processor $i$

The approach for detecting malfunctions in the control law calculations is shown in Figure 1:

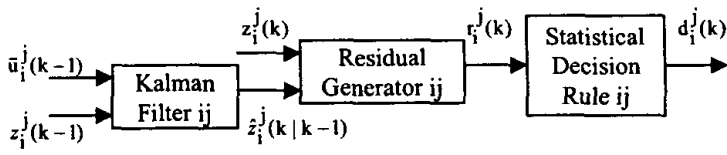

Figure 1: Design Approach for Detecting Malfunctions in Control Law Calculation $j$ of Controller $i$.

The control law calculation $\mathrm{j}$ of the ith controller is monitored using the residual:

$$
\mathrm{r}_{\mathrm{i}}^{\mathrm{j}}(\mathrm{k})=\mathrm{z}_{\mathrm{j}}^{\mathrm{j}}(\mathrm{k})-\hat{z}_{\mathrm{i}}^{\mathrm{j}}(\mathrm{k} \mid \mathrm{k}-1)
$$

where $z_{i}^{j}(k)$ is defined by equation (3), and $\hat{z}_{i}^{j}(k \mid k-1)$ is an estimate of $\mathrm{E}\left[z_{\mathrm{i}}^{\mathrm{j}}(\mathrm{k}) \mid Z_{\mathrm{i}} \mathrm{j}^{\mathrm{k}-1}\right]$ defined for equation (2). The estimate $\hat{z}_{\mathfrak{i}}^{j}(\mathrm{k} \mid \mathrm{k}-1)$ can be produced using a Kalman filter. Under the stated assumptions and using a Gaussian approximation for the conditional density of the measurement under both the nominal and the malfunction hypotheses [3], the Bayesian decision rule can be shown to be:

$$
\begin{aligned}
& \mathrm{d}_{\mathrm{i}}^{\mathrm{j}}(\mathrm{k})=1 \\
& \left|r_{i}^{j}(k)\right| \geq\left|\lambda_{i}^{j}(k)\right| \\
& \mathrm{d}_{\mathrm{i}}^{\mathrm{j}}(\mathrm{k})=-1
\end{aligned}
$$

where the threshold is defined for three cases [3] - [5].

Case 1: $\mathrm{P}_{\mathrm{li}}^{\mathrm{j}}(\mathrm{k})>\mathrm{P}_{0 \mathrm{i}}^{\mathrm{j}}(\mathrm{k})$

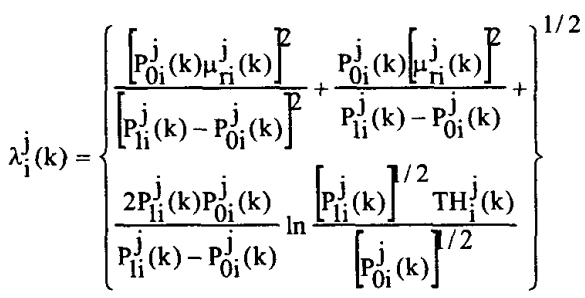

$$
\begin{aligned}
& -\frac{\mathrm{P}_{0 \mathrm{i}}^{\mathrm{j}}(\mathrm{k}) \mu_{r i}^{\mathrm{j}}(\mathrm{k})}{\mathrm{P}_{\mathrm{li}}^{\mathrm{j}}(\mathrm{k})-\mathrm{P}_{0 \mathrm{i}}^{\mathrm{j}}(\mathrm{k})} \\
& \mu_{\mathrm{ri}}^{\mathrm{j}}(\mathrm{k})=\mu_{\mathrm{li}}^{\mathrm{j}}(\mathrm{k})-\mu_{0 \mathrm{i}}^{\mathrm{j}}(\mathrm{k})
\end{aligned}
$$

$\mathrm{P}_{0 \mathrm{i}}^{\mathrm{j}}(\mathrm{k})=$ variance of residual under hypothesis $\mathrm{H}_{0}$ 


$$
\begin{aligned}
& \mathrm{P}_{\mathrm{li}}^{\mathrm{j}}(\mathrm{k})=\text { variance of residual under hypothesis } \mathrm{H}_{1} \\
& \mu_{\mathrm{ri}}^{\mathrm{j}}(\mathrm{k})=\text { mean of residual } \\
& \mu_{\mathrm{li}}^{\mathrm{j}}(\mathrm{k})=\text { mean of residual under hypothesis } \mathrm{H}_{1} \\
& \mu_{0 \mathrm{i}}^{\mathrm{j}}(\mathrm{k})=\text { mean of residual under hypothesis } \mathrm{H}_{0} \\
& \mathrm{TH}_{\mathrm{i}}^{\mathrm{j}}(\mathrm{k})=\text { Bayes Risk Criterion for the detector } \\
& \quad \text { for control law calculation } \mathrm{j} \text { of controller } i
\end{aligned}
$$

$\underline{\text { Case 2: }} \mathrm{P}_{1 \mathrm{i}}^{\mathrm{j}}(\mathrm{k})<\mathrm{P}_{0 \mathrm{i}}^{\mathrm{j}}(\mathrm{k})$

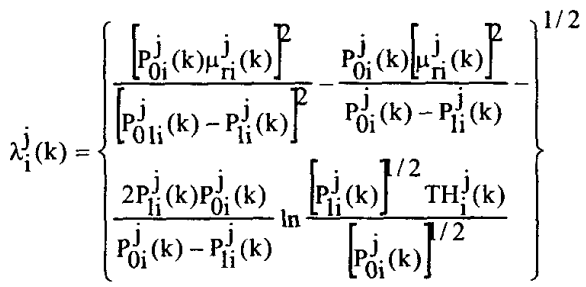

$$
\begin{aligned}
& +\frac{\mathrm{P}_{0 \mathrm{i}}^{\mathrm{j}}(\mathrm{k}) \mu_{\mathrm{ri}}^{j}(\mathrm{k})}{\mathrm{P}_{0 \mathrm{i}}^{\mathrm{j}}(\mathrm{k})-\mathrm{P}_{1 \mathrm{i}}^{\mathrm{j}}(\mathrm{k})}
\end{aligned}
$$

Note that for this case, the inequalities in equation (7) are reversed.

Case 3: $\quad \mathrm{P}_{1 \mathrm{i}}^{\mathrm{j}}(\mathrm{k})=\mathrm{P}_{0 \mathrm{i}}^{\mathrm{j}}(\mathrm{k})$

$$
\lambda_{\mathrm{i}}^{\mathrm{j}}(\mathrm{k})=\frac{\mu_{\mathrm{ri}}^{\mathrm{j}}(\mathrm{k})}{2}+\frac{\mathrm{p}_{0 \mathrm{i}}^{\mathrm{j}}(\mathrm{k}) \ln \left[\mathrm{TH}_{\mathrm{i}}^{\mathrm{j}}(\mathrm{k})\right]}{\mu_{\mathrm{ri}}^{\mathrm{j}}(\mathrm{k})}
$$

The performance of the detector is determined by the probability of a false alarm and the probability of a missed detection. The probability of false alarm is:

$$
\begin{aligned}
& \operatorname{Pfa}_{\mathrm{i}}^{\mathrm{j}}(\mathrm{k})=\mathrm{P}\left[\mathrm{d}_{\mathrm{i}}^{\mathrm{j}}(\mathrm{k})=1 \mid \mathrm{H}_{0}\right] \\
& =\int_{\lambda_{\mathrm{i}}^{\mathrm{j}}(\mathrm{k})}^{\infty} \mathrm{p}_{\mathrm{H}_{0}}\left[\mathrm{z}_{\mathrm{i}}^{\mathrm{j}}(\mathrm{k}) \mid \mathrm{Z}_{\mathrm{i}}^{\mathrm{j}-1}\right] \mathrm{d} z_{\mathrm{i}}^{\mathrm{j}}(\mathrm{k})
\end{aligned}
$$

where $p_{H_{0}}\left[z_{i}^{j}(k) \mid Z_{i}^{j^{k-1}}\right]$ is Gaussian [3] and $\lambda_{j}^{j}(k)$ is the threshold, given by equation $(8 \mathrm{a})-(8 \mathrm{c})$, of the decision rule. The probability of a missed error detection in the $\mathrm{jth}$ calculation of the ith processor is:

$$
\begin{aligned}
& \operatorname{Pm}_{i}^{j}(k)=1-P\left[d_{i}^{j}(k)=1 \mid H_{1}\right] \\
& \left.=1-\int_{\lambda j_{j}^{j}(k)}^{\infty} p_{H_{1}}\left[z_{i}^{j}(k)\right\} Z_{i}^{j k-1}\right] d z_{I}^{J}(k)
\end{aligned}
$$

where $p_{H_{1}}\left[z_{i}^{j}(k) \mid Z_{i}^{j^{k-1}}\right]$ is approximated by a Gaussian density [3] and $\lambda_{i}^{j}(k)$ is the threshold, given by equation (8a) $-(8 c)$, of the decision rule. It can be shown [3], that this approximation yields a conservative detector in the sense that the probability of a missed detection will be lower than that of the detector designed without the Gaussian approximation. However, the probability of false alarm will be higher in this detector.

\subsection{Malfunction Detector for Processor i}

The design approach for detecting malfunctions in the ith processor is illustrated in Figure 2.

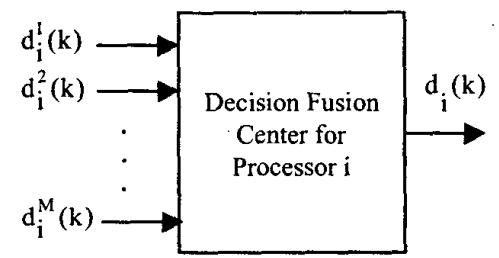

Figure 2: Design Approach for Detecting Malfunctions in Processor i .

The decision $\mathrm{d}_{\mathrm{i}}(\mathrm{k})$ for the ith processor is a fusion of the decisions $\mathrm{d}_{\mathrm{i}}^{\mathrm{j}}(\mathrm{k})[\mathrm{j}=1, \ldots, \mathrm{M}]$ from the detectors for the $\mathrm{M}$ control law calculations of the ith processor. The decision fusion rule for processor $i$ is:

$$
\begin{gathered}
\prod_{j=1}^{M}\left[\frac{1-P m_{i}^{j}(k)}{P a_{i}^{j}(k)}\right]_{i}^{h_{i}^{j}(k)}\left[\frac{P m_{i}^{j}(k)}{1-P f_{i}^{j}(k)}\right]^{\left[1-h_{i}^{j}(k)\right]} \\
d_{i}(k)=1 \\
<\quad T H_{i}(k) \\
d_{i}(k)=0 \\
h_{i}^{j}(k)=\left\{\begin{array}{cc}
1, & d_{i}^{j}(k)=1 \\
0, & d_{i}^{j}(k)=-1
\end{array}\right\}
\end{gathered}
$$


The decision $d_{i}^{j}(k)$ for control law calculation $j$ of the $i$ th processor is the output from the detector shown in Figure 1, $\mathrm{Pfa}_{\mathrm{i}}^{\mathrm{j}}(\mathrm{k})$ is the associated probability of false alarm, $\operatorname{Pm}_{\mathrm{i}}^{\mathrm{j}}(\mathrm{k})$ is the associated probability of a missed detection, and $\mathrm{TH}_{\mathrm{i}}(\mathrm{k})$ is the Bayes Risk Criterion for the detector of the ith processor. The performance of the detector for processor $i$ is determined by the probability of false alarm and the probability of miss, which can be calculated as functions of the performance of the detectors for the $M$ control law calculation error detectors:

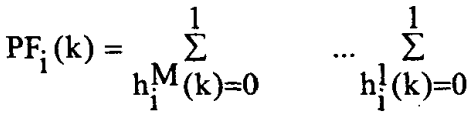

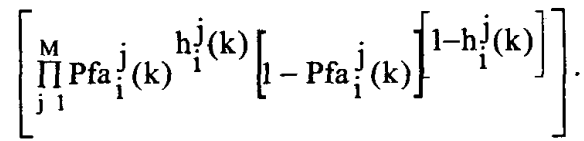

$$
\begin{aligned}
& U\left\{\sum_{j=1}^{M}\left[\frac{1-P m_{i}^{j}(k)}{P a_{i}^{j}(k)}\right]^{h_{i}^{j}(k)} \cdot\left[\frac{P m_{i}^{j}(k)}{1-P a_{i}^{j}(k)}\right]^{\left[1-h_{i}^{j}(k)\right]}-T H_{i}(k)\right\} \\
& \mathrm{PM}_{\mathrm{i}}(\mathrm{k})=1-\mathrm{h}_{\mathrm{i}}^{\sum_{(\mathrm{k})=0}^{1}} \quad \ldots \sum_{\mathrm{h}_{\mathrm{i}}(\mathrm{k})=0}^{1} \\
& {\left[\prod_{j}^{M}\left[1-P m_{i}^{j}(k)\right]^{h_{i}^{j}(k)} \operatorname{Pm}_{i}^{j} j_{(k)}^{\left[1-h_{i}^{j}(k)\right]}\right]} \\
& \left.U\left\{\mathrm{M}\left[\frac{1-P m_{i}^{j}(k)}{P f_{i}^{j}(k)}\right]^{h_{i}^{j}(k)}\left[\frac{P m_{i}^{j}(k)}{1-P f_{i}^{j}(k)}\right]^{\left[1-h_{i}^{j}(k)\right.}\right]-T H_{i}(k)\right\}
\end{aligned}
$$

where $U\left\{{ }^{*}\right\}$ is the unit step function.

\subsection{Malfunction Detector for the Controller}

The design approach for detecting malfunctions in the fault tolerant controller is exactly analogous to that for the ith processor and is illustrated in Figure 3.

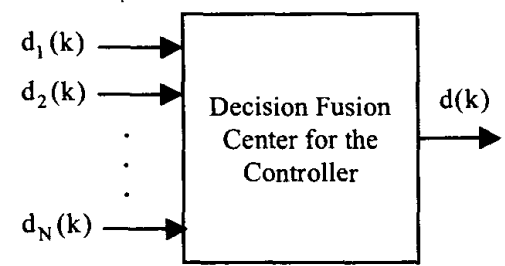

Figure 3: Design Approach for Detecting Malfunctions in the Fault Tolerant Controller.
The decision $d(k)$ is a fusion of the decisions $d_{j}(k)[i=1$, . .., N] from the detectors for the $\mathrm{N}$ processors. The decision fusion rule for the controller is:

$$
\begin{aligned}
\prod_{i=1}^{N}\left[\frac{1-P M_{i}(k)}{P F_{i}(k)}\right]^{d_{i}(k)} & {\left[\frac{P M_{i}(k)}{1-P F_{i}(k)}\right]^{\left[1-d_{i}(k)\right]} } \\
d(k) & =1 \\
\geq & T H(k) \\
< & \\
d(k) & =0
\end{aligned}
$$

This decision fusion rule is based on the decision, $\mathrm{d}_{\mathrm{i}}(\mathrm{k})$, from the detector for each of the $\mathrm{N}$ processors and its associated performance, as characterized by the probability of false alarm $\mathrm{PF}_{\mathrm{i}}(\mathrm{k})$ and the probability of a missed detection $\mathrm{PM}_{\mathrm{i}}(\mathrm{k})$. The threshold is the Bayes Risk Criterion for the detector for the controller. The performance of the detector for the controller is determined by the probabilities of false alarm and missed detection, which can be calculated as functions of the performance probabilities of the detectors for each of the processors:

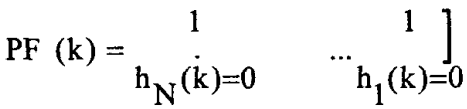

$$
\begin{aligned}
& {\left[\prod_{i=1}^{N} P F_{i}(k)^{d_{i}(k)} 1-P F_{i}(k)\left[1-d_{i}(k)\right]\right] \text {. }} \\
& U\left\{{ }_{i=1}^{N}\left[\frac{1-P M_{i}(k)}{P F_{i}(k)}\right]^{d_{i}(k)}\left[\frac{P M_{i}(k)}{1-P F_{i}(k)}\right]^{\left[1-d_{i}(k)\right]}-T H(k)\right\}
\end{aligned}
$$

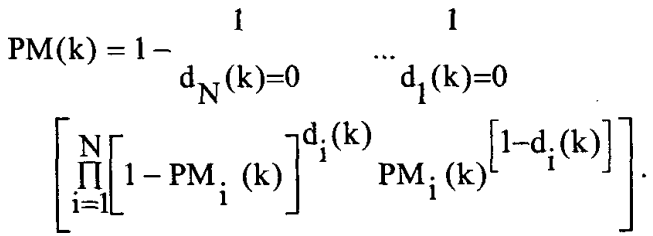

$$
\begin{aligned}
& U\left\{N\left[\frac{1-P_{i}(k)}{P F_{i}(k)}\right]^{d_{i}(k)}\left[\frac{P M_{i}(k)}{1-P F_{i}(k)}\right]^{\left[1-d_{i}(k)\right]}-T H(k)\right\}
\end{aligned}
$$

where $U\left\{^{*}\right\}$ is the unit step function.

\subsection{Demonstration Using Experimental Data}

The monitoring strategy of Section 3 is demonstrated for the elevator and throttle commands of a B737 Autoland 
flight controller using experimental data. Closed-loop laboratory experiments were performed at the NASA Langley Research Center in which a quad-redundant flight control computer was exposed to HIRF while executing a simulated approach and landing. During these experiments, the flight control computer was executing the Autoland function inside the HIRF test chamber while operating in closed-loop with a computer simulation of the aircraft and heavy clear air turbulence ( 20 knot steady winds, $6 \mathrm{ft} / \mathrm{s}$ gusts from NE at 45 degrees). Electrical isolation between the controller and the simulation computer was achieved by converting all electrical signals to optical signals. Figure 4 shows a photograph of the quad-redundant flight control computer in the HIRF chamber during testing.

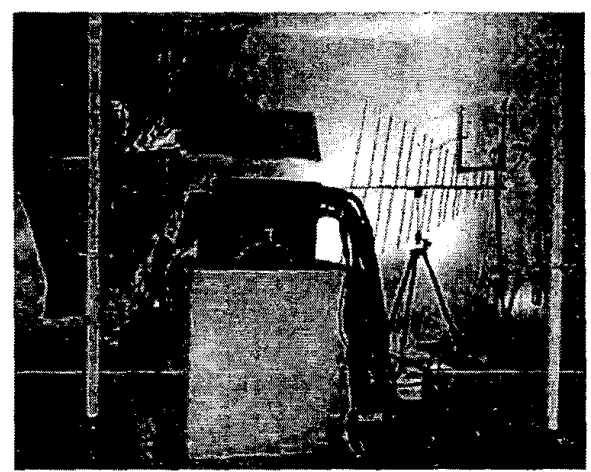

Figure 4: Quad-Redundant Flight Control Computer in NASA Langley HIRF Chamber.

In this example, $\mathrm{N}=4$ processors and $\mathrm{M}=2$ command calculations per processor. The data frame rate for each run is $50 \mathrm{~ms}$ per frame. Two sets of data were used in this example. The first set contained 51 runs with no HIRF exposure, and the second set contained 24 runs with constant HIRF continuous wave exposure at $550 \mathrm{MHz}$, $600 \mathrm{~V} / \mathrm{m}$. The state-space model was obtained using a single run from the data set with no HIRF. The mean and variance of the residual under hypothesis $\mathrm{H}_{0}$ were determined using the remaining 50 runs of the data set with no HIRF. The mean and variance of the residual under hypothesis $\mathrm{H}_{1}$ were determined using the 24 runs with HIRF exposure. All detectors were designed to make a decision at each data frame based on a single observation. The Bayes Risk Criterion for all detectors was set equal to 1. This corresponds to zero costs for correct decisions, equal costs for incorrect decisions, and equal a priori probabilities for the two hypotheses. The state-space models were obtained using parameter identification methods developed at NASA Langley [13] - [14].

The performance of the detector for elevator command error in processor 1 of the quad-controller is shown in Figures 5 and 6.

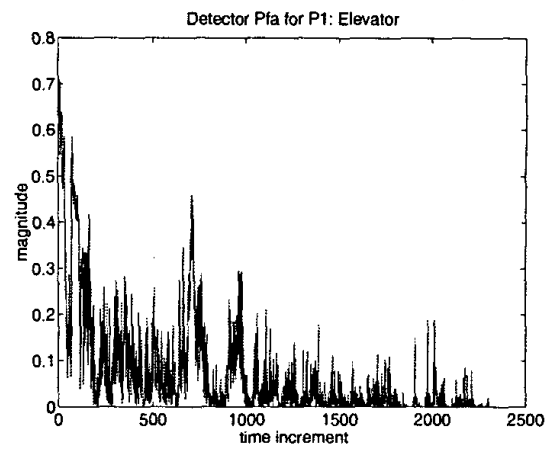

Figure 5: Probability of False Alarm for the Elevator Command Error Detector for Processor 1

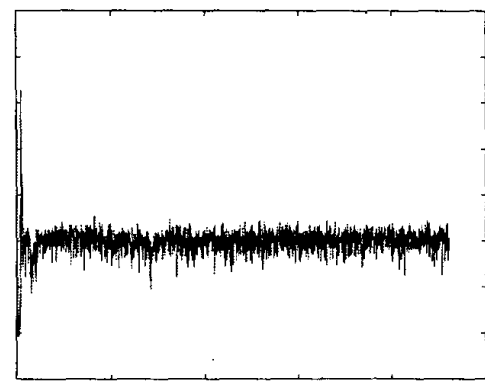

Figure 6: Probability of Miss for the Elevator Command Error Detector for Processor 1

The performance for this detector is relatively poor, varying for each time step. However, there are data frames is which the probability of miss is approximately 0.45 and the probability of false alarm is relatively low. Note that correct detection at each frame may not be necessary for this application due to slow vehicle dynamic response relative to the $50 \mathrm{~ms}$ frame rate. The performance of the detector for throttle command errors in processor 1 is shown in Figures 7 and 8.

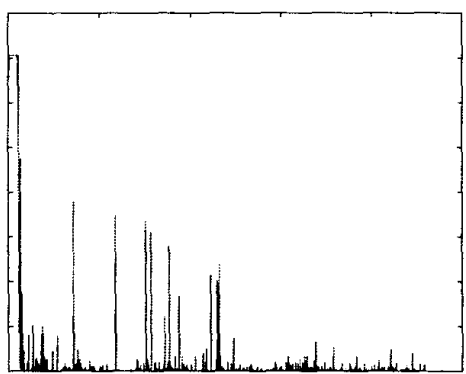

Figure 7: Probability of False Alarm for the Throttle Command Error Detector for Processor 1 


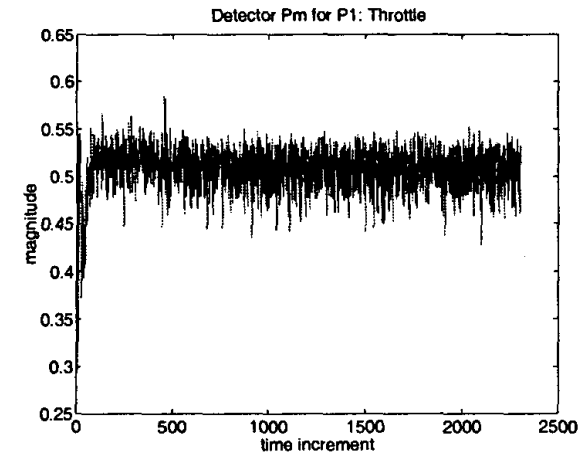

Figure 8: Probability of Miss for the Throttle Command Error Detector for Processor 1

The performance of this detector is similar to that for the elevator error detector. There are time steps at which the probability of miss is approximately 0.45 and the probability of false alarm is relatively low. The performance of the detectors for the throttle and elevator commands of the other three processors was comparable to that for processor 1 .

The detector for processor 1 is comprised of the fusion center for the decisions from the throttle and elevator error detectors of processor 1 at each time step. The performance of the detector for processor 1 is shown is Figures 9 and 10.

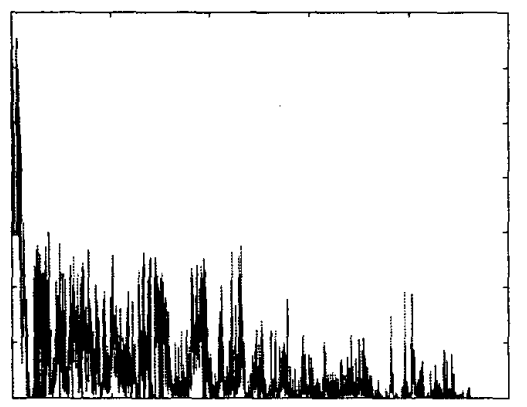

Figure 9: Probability of False Alarm for the Error Detector for Processor 1

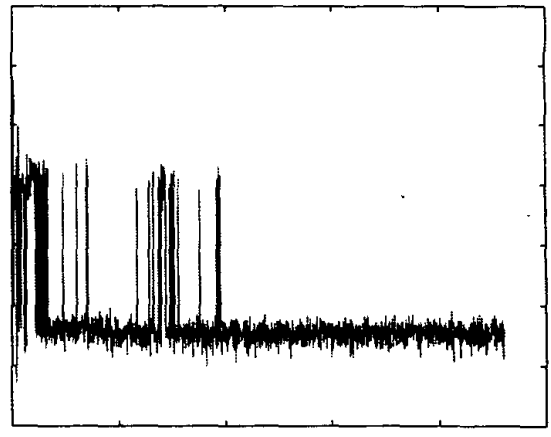

Figure 10: Probability of Miss for the Error Detector for Processor 1

The performance of the error detector for processor 1 is generally better than the local detectors for processor 1 . False alarm probability is still less than 0.2 while the missed detection probability is now less than 0.3 for most time steps. The performance for the error detectors for the other three processors was comparable to that for processor 1 .

The error detector for the controller is the fusion center for the error decisions for the four processors. The performance of the error detector for the controller is shown in Figures 11 and 12.

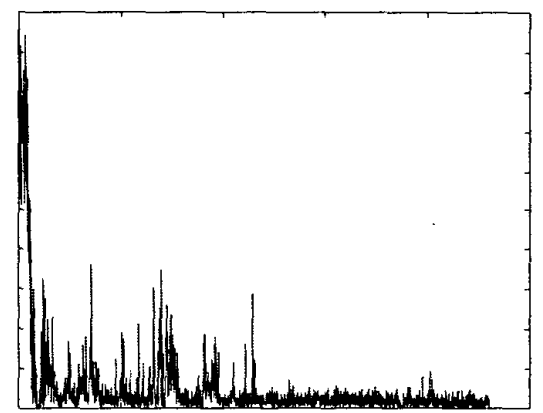

Figure 11: Probability of False Alarm for the Error Detector for the Controller 


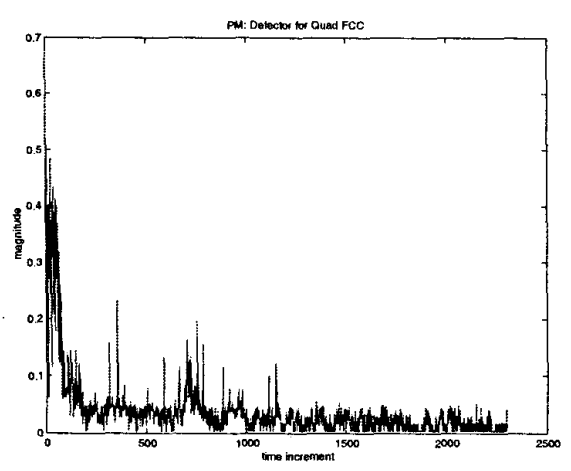

Figure 12: Probability of Miss for the Error Detector for
Controller

The detector for the controller has a probability of detection that is better than 0.9 for most time steps and a probability of false alarm that is better than 0.05 for most time steps. This substantial performance improvement may be indicative of the value of a distributed detection approach using data fusion techniques.

\subsection{Summary and Conclusions}

A general approach has been presented using distributed detection and data fusion techniques to detect malfunctions in a fault tolerant control computer, and in any one of the individual processors. Malfunction in the controller is detected by fusing the decisions from the error detectors for $\mathrm{N}$ processors. Malfunction in each processor is detected by fusing the decisions from the $\mathrm{M}$ control law error detectors. The optimal fusion rules for each of these detectors is a function of the decisions from the detectors of the previous stage and the associated detector performance. The fusion rule for detecting malfunctions in the processors requires the performance of the error detectors for the control law calculations. The methodology for monitoring control integrity that was developed in this paper is limited by the stated assumptions. The independence assumptions for malfunctions in the control laws and for each of the data fusion problems idealize conditions that could occur. However, these assumptions greatly simplify the data fusion problem because values of correlation coefficients do not have to be determined.

The multi-stage distributed detection and data fusion scheme was implemented for the first time using experimental data collected during HIRF exposure experiments on a quad-redundant flight control computer. In these experiments, the controller was configured as a B737 Autoland system. Flight conditions for the simulated approach and landing included severe turbulence. Detectors were designed for the throttle and elevator commands for each processor. These detectors required a mathematical characterization of complex nonlinear control law calculations under flight conditions with heavy clear air turbulence. While the performance of these detectors was relatively poor, overall detection performance for controller errors was significantly improved using the proposed distributed detection and decision fusion scheme.

The performance of the controller error detector could be improved by using a better design for the control law calculation error detectors. Future work includes: i) development of improved designs for the control law calculation error detectors, ii) implementation and demonstration of this design in the laboratory; and iii) removal of independence assumptions for a redesign of the monitor to account for correlation between observations.

\section{REFERENCES}

1. Belcastro, C. M., "Closed-Loop HIRF Experiments Performed on a Fault Tolerant Flight Control Computer", Proceedings of the Digital Avionics Systems Conference, Irvine CA, October 1997

2. Belcastro, C. M., "Ensuring Control Integrity of Critical Systems Subjected to Electromagnetic Disturbances: Problem Overview", Proceedings of the American Control Conference", Philadelphia PA, June 1998

3. Belcastro, C. M., "Detecting Upset in Fault Tolerant Control Computers Using Data Fusion Techniques", Ph.D Dissertation, Drexel University, December 1994

4. Belcastro, C. M., Fischl, R. F., "Monitoring Functional Integrity in Fault Tolerant Aircraft Control Computers for Critical Applications", Proceedings of the International Federation of Automatic Control, San Francisco CA, June 1996

5. Belcastro, C. M., "Detecting Controller Malfunctions in Electromagnetic Environments: Part II - Design \& Simulation of the Detector", Proceedings of the Conference on Control Applications, Hawaii, 1999

6. Belcastro, C. M., "Monitoring Functional Integrity in Critical Control Computers Subjected to Electromagnetic Disturbances", Proceedings of the American Control Conference, Philadelphia PA, June 1998

7. Eure, K., W., "Fault Detection of a Flight Control Computer in a Harsh Electromagnetic Environment", Proceedings of the Digital Avionics Systems Conference, Daytona Beach, Florida, October 2001

8. W. S. Gray, O. R. González and M. Dogan, "Stochastic Perturbation Models of Electromagnetic Disturbances in Closed-Loop Computer Controlled Flight Systems", 1999 Digital Avionics Systems Conference, St. Louis, MO, 1999

9. O. R. González, W. S. Gray and S. Patilkulkarni, "Analysis of Memory Bit Errors Induced by Electromagnetic Interference in Closed-Loop Digital Flight Control Systems", Digital Avionics Systems Conference, Philadelphia, PA, 2000

10. O. R. González, W. S. Gray and A. Tejada, "Analysis of Design Tradeoffs in Rollback Recovery Methods for Fault Tolerant Digital Control Systems", Proc. 2002 American Control Conference, Alaska, 2002

11. Robust Model-Based Fault Diagnosis for Dynamic Systems, Chen \& Patton, Kluwer Academic Publishers, 1999

12. Distributed Detection and Data Fusion, Varshney, Springer, 1997

13. Juang, J-N., and Phan, M., "Identification of System, Observer, and Controller from Closed-Loop Experimental Data," Proceedings of AIAA Guidance, Navigation and Control Conference, Hilton Head, SC, 1992

14. Applied System Identification, Juang, Prectice Hall, 1994 